RELATO DE CASOS

\title{
Angiodisplasia do Ceco na Terceira Década de Vida ${ }^{1}$
}

\author{
Angiodysplasia of the Cecum in the Third Decade of Life
}

\author{
NELSON FONTANA MARGARIDO ${ }^{1}$, HORÁCIOCONSOLMAGNO ${ }^{1}$, LUIZ PICCININI FILHO ${ }^{2}$, DENISE GONÇALVES \\ PRIOLLI $^{3}$, CARLOS AUGUSTOREAL MARTINEZ ${ }^{3}$ \\ ${ }^{1}$ Departamento de Cirurgia da Faculdade de Medicina da Universidade de São Paulo (FMUSP), São Paulo; \\ ${ }^{2}$ Faculdade de Ciências Médicas da Santa Casa de São Paulo (FCMSC). Hospital Santa Isabel, São Paulo; ${ }^{3}$ Disciplina \\ de Cirurgia Geral do Curso de Medicina da Universidade São Francisco (USF), Bragança Paulista, São Paulo.
}

MARGARIDO NF; CONSOLMAGNO H; PICCININI FILHO L; PRIOLLI DG; MARTINEZ CAR. Angiodisplasia do Ceco na Terceira Década de Vida. Rev bras Coloproct, 2007;27(2): 202-206.

RESUMO: As malformações vasculares representam uma das principais causa de hemorragia digestiva baixa em doentes idosos. $O$ acometimento de indivíduos com menos de trinta anos é evento raramente descrito. Objetivo: O objetivo do presente relato é apresentar um caso de angiodisplasia, localizada na região cecal, em doente na terceira década de vida, diagnosticada por meio de arteriografia pré-operatória e confirmada pelo estudo anátomo-patológico. Relato do caso: Homem, 29 anos, com queixa de anemia crônica de origem obscura há vários anos e refratária ao tratamento clínico e transfusional. Com o objetivo de investigar possível origem digestiva foi submetido à arteriografia seletiva abdominal que demonstrou a presença de malformação vascular, localizada na região cecal. Realizou-se colectomia direita onde foi possível confirmar a localização da enfermidade pela arteriografia pós-operatória do espécime extirpado e o diagnóstico definitivo de angiodisplasia pelo estudo anátomo-patológico. Após a intervenção cirúrgica apresentou evolução favorável, estando atualmente no $5^{\circ}$ mês pós-operatório sem apresentar sinais de recidiva da anemia. Conclusão: Não obstante à raridade, a possibilidade de angiodisplasia deverá ser sempre considerada na investigação da anemia de causa obscura em doentes jovens.

Descritores: Angiodisplasia. Malformações arteriovenosas. Doenças do Ceco. Cirurgia Colorretal. Cólon.

\section{INTRODUÇÃO}

Angiodisplasias são lesões vasculares degenerativas, adquiridas, geralmente encontradas na parede intestinal de pacientes idosos. ${ }^{1}$ Estas malformações vasculares caracterizam-se pela presença de dilatações tortuosas do plexo vascular submucoso que, ao sofrerem rupturas espontâneas, provocam sangramento para o interior da luz intestinal. ${ }^{2} \mathrm{~A}$ enfermidade representa uma das principais causas de hemorragia digestiva baixa em indivíduos entre a sexta e sétima décadas de vida, surgindo com maior freqüência no ceco e cólon ascendente. $\mathrm{O}$ acometimento de adultos jovens é excepcional e raramente descrito. ${ }^{2,3}$
O objetivo do presente relato é descrever um caso de angiodisplasia do ceco, em doente com menos de trinta anos de idade, diagnosticada por angiografia abdominal e tratada com sucesso por meio da ressecção cirúrgica.

\section{RELATO DO CASO}

Homem, branco, 29 anos, realizando há dois anos, tratamento clínico para anemia crônica e não apresentando respostas sustentadas adequadas aos tratamentos medicamentosos instituídos, necessitando de transfusões sangüíneas periódicas. Ao exame cardiológico não havia sinais de estenose aórtica. Com o objetivo de investigar possível perda digestiva foi sub-

Trabalho realizado na Disciplina de Técnica Cirúrgica da Faculdade de Medicina da Universidade de São Paulo, São Paulo, Brasil.

Recebido em 08/09/2006

Aceito para publicação em 16/01/2007 
metido à endoscopia digestiva alta e colonoscopia que se revelaram normais. Como a pesquisa de sangue oculto nas fezes mostrava-se constantemente positiva, foi submetido a arteriografia seletiva dos troncos celíaco e mesentéricos que demonstrou, a partir do ramo ileocólico da artéria mesentérica superior, segmento de alça intestinal que apresentava significativo aumento no número de vasos do plexo sangüíneo submucoso. Observou-se ainda que nesse local os vasos sangüíneos eram irregulares, agrupados, tortuosos e de maior calibre que em outros segmentos da parede intestinal. A drenagem venosa desse território ocorria mais precocemente, contrastando veias de maior calibre. Não se identificaram pontos de extravasamento de contraste para a luz intestinal em todos os territórios estudados. O resultado do exame permitiu o diagnóstico de malformação vascular em topografia do ceco e segmento proximal do cólon direito. (Figura 1)

Diante do achado angiográfico, bem como das infrutíferas tentativas clínicas de correção da anemia optou-se pelo tratamento cirúrgico, tendo sido realizado colectomia direita por via convencional.

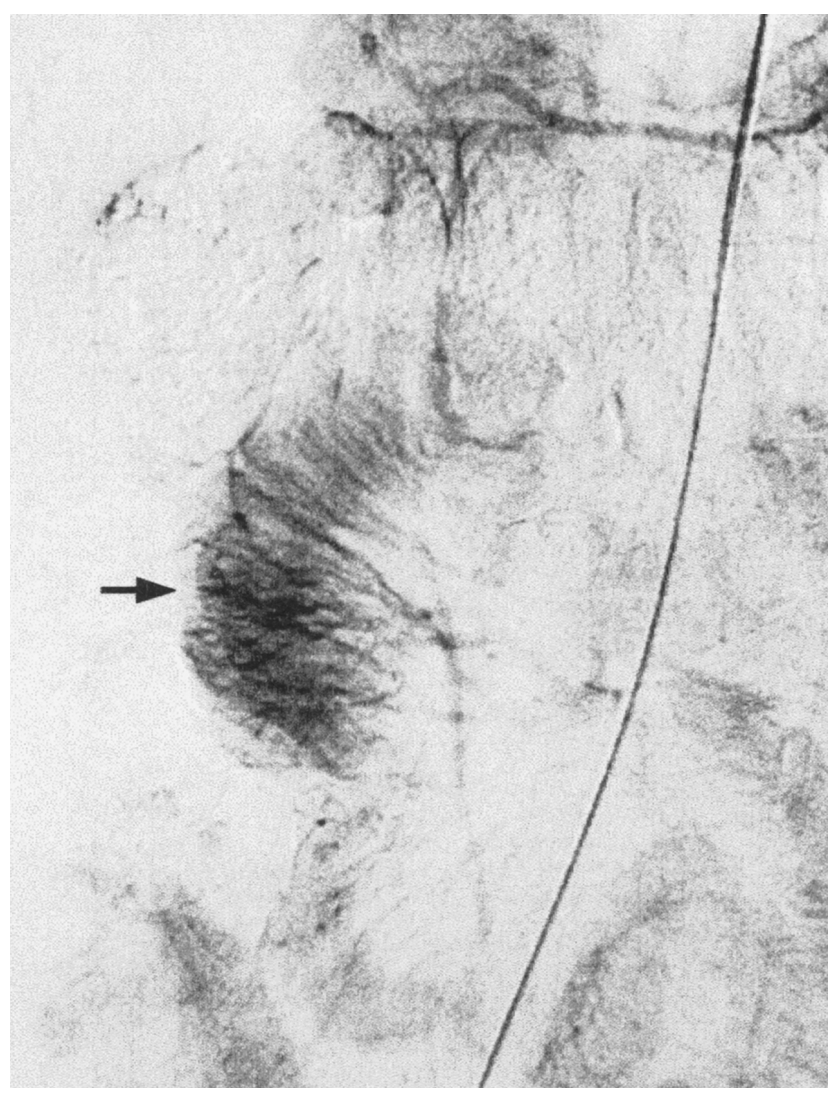

Figura 1 - Imagem de arteriografia abdominal. Aumento localizado do plexo sanguíneo submucoso (seta).
Imediatamente após a extirpação do espécime cirúrgico, realizou-se a arteriografia através da infusão de contraste iodado pela artéria íleo-ceco-cólica, constatando-se que no segmento extirpado encontravamse as mesmas alterações vasculares verificadas na arteriografia pré-operatória. (Figura 2).

O estudo histopatológico do espécime ressecado demonstrou a presença de dilatação e congestão de vasos do plexo sangüíneo submucoso que eram tortuosos e atingiam a camada muscular da mucosa. Não se identificaram erosões da mucosa cólica, que apresentava pequenas áreas com infiltrado inflamatório. Diante do achado foi possível firmar o diagnóstico de angiodisplasia do ceco. (Figura 3).

Após a intervenção cirúrgica o paciente apresentou boa evolução, tendo recebido alta hospitalar no

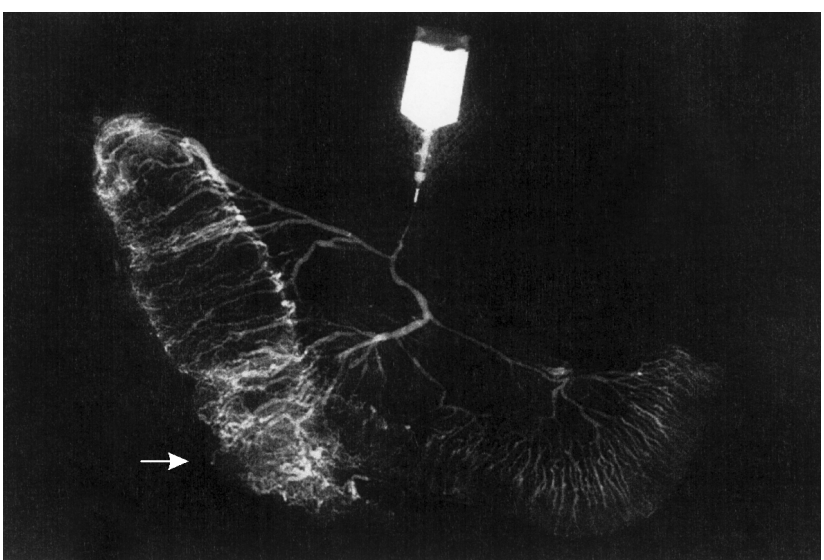

Figura 2 - Arteriografia pós-operatória do espécime cirúrgico, onde se observa acentuado conjunto de vasos mais calibrosos na região do cecal, quando comparado com os demais segmentos ressecados (seta).

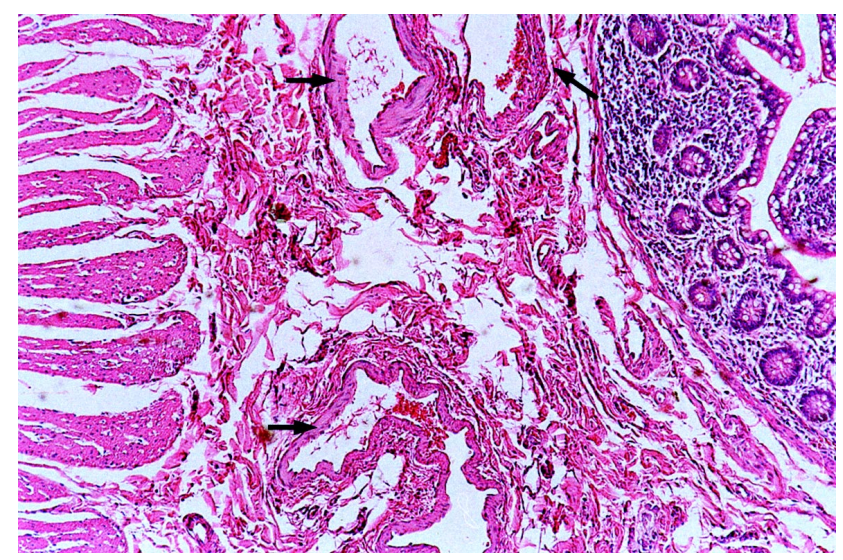

Figura 3 - Exame histopatológico do espécime extirpado mostrando ectasias vasculares (setas) na camada submucosa da parede intestinal. (H.E.-100x). 
$3^{\circ}$ pós-operatório. Atualmente, encontra-se no $5^{\circ}$ mês de seguimento, sem sinais clínicos ou laboratoriais de anemia não mais necessitando de transfusões sangüíneas.

\section{DISCUSSÃO}

A primeira referência encontrada na literatura de hemorragia digestiva baixa oriunda de malformações vasculares localizadas no intestino grosso foi feita em $1839^{4}$. A partir desta descrição inicial, outros casos foram publicados, cabendo a Galdabini e cols em $1978^{5}$ o emprego do termo angiodisplasia para descrever malformações vasculares encontradas na parede intestinal. Desde então, muita confusão passou a existir para a correta nomenclatura destas alterações vasculares, e sinônimos como hemangiomas, telangiectasias, e ectasias vasculares passaram a ser adotado para descreverem a enfermidade 4 . Tal confusão decorre em parte da multiplicidade de anomalias vasculares que podem acometer o trato digestivo, pois, embora possuam diferentes etiologias exteriorizam-se clinicamente por sangramento digestivo baixo. Com o intuito de padronizar a nomenclatura, Camilleri e cols em $1984^{6}$, apresentaram uma proposta de classificação baseada no tamanho e tipo dos vasos sangüíneos comprometidos e a concomitância com alterações clínicas ou dermatológicas.

A angiodisplasia atinge ambos os sexos em igual proporção ocorrendo predominantemente em doentes entre 60 e 70 anos de idade. $\mathrm{O}$ acometimento de indivíduos jovens, a semelhança do caso descrito no presente relato, é achado excepcional. ${ }^{7,8}$ Os doentes geralmente queixam-se de um ou mais episódios de sangramento digestivo baixo, algumas vezes de grande monta. Muitos enfermos evoluem com anemia crônica decorrente de perdas sanguiíneas detectáveis apenas pela pesquisa de sangue oculto nas fezes, sem apresentarem quadro de hemorragia digestiva franca ${ }^{4}$.

A maior prevalência da angiodisplasia em pacientes com idades avançadas (em geral com mais de 60 anos) fez supor que provavelmente se tratem de lesões degenerativas relacionadas ao processo de envelhecimento $^{9,10}$. Boley e cols ${ }^{9}$. acreditam que as alterações vasculares são de origem adquirida causadas diretamente por obstrução gradual e intermitente de veias submucosas, principalmente onde transpõe as camadas musculares da parede cólica. Com o passar dos anos essas obstruções promoveriam aumento da pressão no interior dos vasos levando a dilatação de capilares e a formação de comunicações arteriovenosas ${ }^{9}$. Os autores encontraram ectasias mucosas e dilatação de veias da camada submucosa em 27 e $53 \%$ respectivamente em 15 doentes que nunca haviam apresentado sangramento intestinal. Tais evidências ficam reforçadas por um estudo com 52 indivíduos assintomáticos, com média de idade de 75 anos (34-90 anos), que demonstrou no exame necroscópico a presença de angiodisplasias em 26 (50\%) oportunidades ${ }^{11}$.

As alterações vasculares do conjunto das túnicas mucosa e submucosa parece decorrerem da dilatação e tortuosidade progressivas dos vasos, e não por malformações congênitas. A contínua e repetida distensão e contração do cólon, além de provocar a dilatação do plexo sangüíneo também ocasionaria aumento da pressão no interior dos vasos aumentando a possibilidade de sangramento ${ }^{10,12}$. Reforçam estas evidências a localização preferencial das angiodisplasias na região cecal, justamente o segmento do intestino grosso que possui maior diâmetro o que determina, ao longo do tempo, maior tensão em sua parede. Estas observações encontram subsídio quando se considera a lei de Laplace que determina quanto maior o raio do vaso, maior a tensão na parede requerida para resistir uma dada pressão interna de fluido. ${ }^{13}$ Cabe destacar que a simples presença dessas ectasias não provoca, necessariamente, sangramento digestivo fazendo com que um grande número de pacientes sejam assintomáticos.

A associação das malformações vasculares com estenose aórtica tem suscitado grande discussão a respeito do papel desempenhado pela valvulopatia na etiologia da enfermidade ${ }^{4}$. Alguns autores acreditam que nos portadores de estenose aórtica, o desenvolvimento da displasia vascular seria decorrente da menor perfusão sangüínea na parede intestinal, secundária a redução do débito ventricular esquerdo ${ }^{14}$. Outros acreditam que a menor perfusão sangüínea tecidual, decorrente do menor débito cardíaco, não seria suficiente para o desenvolvimento da malformação, mas poderia aumentar o risco de sangramento por provocarem lesão isquêmica ao endotélio vascular de enfermos que já apresentavam previamente a angiodisplasia ${ }^{9}$. O doente descrito no presente relato, não obstante ser portador da angiodisplasia, não apresentava sinais ou sintomas sugestivos de estenose aórtica.

$\mathrm{Na}$ literatura, a maioria dos estudos se limita a análise histológica do trajeto, tortuosidade e dilatação dos vasos de pequeno calibre, dedicando pouca atenção a quantidade dos vasos encontrados em um segmento es- 
pecífico da parede intestinal. Já foi demonstrado que a média da contagem de vasos de pequeno calibre (vasos com diâmetro de até 40 micrômetros) no conjunto das túnicas mucosa e submucosa de seres humanos em 10 diferentes campos de observação, caracteriza a chamada "densidade linear vascular" ${ }^{13}$. Com o progredir da idade e principalmente a partir da quinta década de vida, existe um aumento proporcional e progressivo, da "densidade linear vascular" nas camadas mucosa e submucosa ${ }^{13}$. A partir destas evidências foi possível criar para seres humanos uma equação de regressão, tendo como variáveis consideradas, o número de vasos encontrados e a idade em anos. Quando esses cálculos são transpostos e aplicados para pacientes na $3^{\mathrm{a}}$ e $4^{\mathrm{a}}$ décadas de vida, o valor da "densidade linear vascular" é extremamente diminuto, sugerindo uma etiologia congênita quando essas malformações são encontradas em indivíduos jovens ou mesmo de meia idade ${ }^{13}$.

Nos doentes idosos a presença de modificações degenerativas intermitentes, alterações pressóricas, dilatações e tortuosidades dos vasos, hipoxemia crônica na microcirculação, além da possibilidade da associação de doenças cardiocirculatórias e ou pulmonares, poderiam explicar a etiologia dessas ectasias vasculares ${ }^{8,12,15,16}$. Todas essas situações também podem ocorrer em adultos jovens, mas com certeza o tempo de existência das mesmas seria insuficiente para determinar o aparecimento das angiodisplasias, reforçando a possibilidade de tratarse de enfermidade congênita ${ }^{13}$. Todos esses argumentos que apóiam teoria de uma enfermidade com características degenerativas dificilmente poderiam ser atribuídos ao doente do presente relato, uma vez que antes dos 29 anos de idade, já apresentava perda sangüínea constante pelo trato digestivo baixo.
O diagnóstico da malformação vascular, na maioria das vezes, é realizado durante o exame endoscópico. Contudo, em algumas oportunidades, fica evidente a importância da realização da arteriografia pré-operatória que demonstra utilidade não só para o diagnóstico da doença, bem como na sua exata localização (íleo terminal, ceco e segmento proximal do cólon direito). A arteriografia assume maior importância nos enfermos com sangramento ativo, conferindo segurança à indicação do tratamento cirúrgico. Nos doentes que não apresentam sangramento ativo, embora o exame demonstre menor acuidade diagnóstica permite identificar corretamente a lesão. A arteriografia pode ainda se revelar uma alternativa terapêutica por permitir a infusão de drogas vasopressoras ou a embolização seletiva arterial no ponto de sangramento ${ }^{4}$.

A arteriografia da peça cirúrgica realizada imediatamente após a ressecção cirúrgica, é exame de fácil execução e acessível à maioria dos centros ${ }^{2,9,17}$. Em virtude das angiodisplasias não serem visíveis ou palpáveis pela superfície serosa da parede intestinal, bem como por apresentarem geralmente diminutas dimensões, sua correta identificação durante a cirurgia poderá ser problemática. A realização imediata de arteriografia no espécime cirúrgico extirpado confere ao cirurgião a certeza da presença da malformação vascular no segmento ressecado. No doente do presente relato, a angiografia do espécime cirúrgico demonstrou, com clareza até mesmo superior a arteriografia pré-operatória, a presença da angiodisplasia.

O acompanhamento do caso descrito no presente relato demonstra que as malformações vasculares, não obstante apresentarem baixa incidência, devem ser sempre consideradas na investigação de anemia de causa obscura em doentes jovens.

ABSTRACT: The vascular malformations represent one of the main causes of lower gastrointestinal bleeding in elderly patients. The incidence of the disease in patients with less than thirty years is a rare described event. The objective of the present report is to show a case of angiodysplasia, located in the cecum region, in-patient on his third decade of life, revealed by preoperative selective angiography and confirmed by histopathological study. Men, $2^{\text {th }}$ years old, presented complaining a chronic anemia of obscure origin for several years and refractory to the clinical and transfusion therapy. With the objective to investigate a possible digestive origin he was submitted to the abdominal selective angiography that demonstrated the presence of vascular malformation located in the cecum. A right colectomy was done and then it was possible to confirm the localization of the disease for postoperative angiography of surgical specimen and the diagnosis of angiodysplasia for the anatomopathologic study. After surgical procedure presented favorable evolution, being currently in the $5^{\text {th }}$ postoperative month without presenting signals of anemia. Despite of an uncommon possibility the presence of angiodysplasia has to be always considered in the clinical investigation of anemia of obscure cause in young patients.

Key words: Angiodysplasia. Arteriovenous malformations. Cecal Diseases. Cecum. Colorectal Surgery. Colon. 


\section{REFERÊNCIAS}

1. Ress HC, Wright NA. Angiodysplasia of the colon. In: Anthony PP, Mc Sween RNM eds. Recent advances in histophatology. Vol 12.Edinburgh: Churchill Linvingstone 1984: 178-88.

2. Segal R, Yogev R,Witz E, Reif R, Orda R. Angiodysplasia of the colon. J R Soc Med.1987;80:249-51.

3. Sharma, R.; Gorbien, M. - Angiodysplasia and lower gastrointestinal tract bleeding in elderly patient. Arch Intern Med.1995;155:807-12.

4. Kheterpal S. Angiodysplasia: a review. J R Soc Med.1991;84:615-8.

5. Galdabini JJ, Waitman AC, Norellio PA, Greenfield AJ, Ezreleta M. Angiodysplasia of the colon: a cause of rectal bleeding. J Cardiovasc Radiol.1978;1:3-13.

6. Camilleri M, Chadwick VS, Hodgson HJ. Vascular anomalies of the gastrointestinal tract. Hepatogastroenterology. 1984;31:149-53.

7. Moore JD, Thompson NW, Appelman HD, Foley D. Arteriovenous malformations of the gastrointestinal tract. Arch Surg.1976;111:381-9.

8. Hemingway A, Allison DJ. Angiodysplasia and Meckel's diverticulum: a congenital association? Br J Surg.1982;69:493-6.

9. BoIey SJ; Sammartano R, Adams A, DiBiase A, Kleinhaus S, Sprayregen S. On the nature and etiology of vascular ectasias of the colon. Degenerative lesions of aging. Gastroenterology. 1977;72:650-60.

10. Boley SJ, Sprayregen S, Sammartano RJ, Adams A, Kleinhaus $\mathrm{S}$. The pathophysiologic basis for the angiographic signs of vascular ectasias of the colon. Radiology.1977;125:615-21.
11. Sabanathan $\mathrm{S}, \mathrm{Nag} \mathrm{S}$. Angiodysplasia of the colon: a postmorten study. J R Coll Surg Edinb.1982;27:285-9.

12. Nuabamm M, Baum B, Blakemore W. Demonstration of intraabdominal bleeding by selective arteriography. JAMA.1965;191:389-90.

13. Teixeira MCR, Margarido NF, Rodrigues C, Rodrigues Junior AJ. Tolosa EMC. Estudo da angioarquitetura dos vasos sangüíneos do ceco humano a partir da $5^{\text {a }}$. década de vida. Rev Col Bras Cir.1997; 5:341-6.

14. Greenstein RJ, McElthnney AJ, Reuben D, Greenstein AJ. Colonic vascular ectasias and aortic stenosis: coincidence or causal relationship? Am J Surg.1986;151:347-51.

15. Carella G, Netri G, Loddo A, Fioravanti PM, Coco C, Bevilacqua E. Angiodysplasia of the colon. Clin Ter.1986;119: 323-9.

16. Rogers BHG. Endoscopic diagnosis and therapy of mucosal vascular abnormalities of the gastrointestinal tract occurring in elderly patients and associated with cardiac, vascular and pulmonary disease. Gastrointest Endosc. 1980; 26:134-8.

17. Pounder DJ, Rowland R, Pieterse AS, Freeman R, Hunter R. Angiodysplasia of the colon. J Clin Pathol.1982;35:824-9.

\section{Endereço para correspondência:}

DR. CARLOS AUGUSTO REAL MARTINEZ

Rua Rui Barbosa, 255 apto.32

Vila Boa Vista - CEP: 09190-370

Santo André - São Paulo - Brasil

Fone: (011) 4438-9203 